\title{
Comment on 'Efficacy of stereotactic body radiotherapy in oligorecurrent and in oligoprogressive prostate cancer: new evidence from a multicentric study'
} Isabel Bravo*,1

\author{
${ }^{1}$ Medical Physics and Radiobiology Group, IPO Porto Research Centre, Portuguese Oncology Institute of Porto, Porto 4200-072, Portugal
}

Sir,

We have read with great interest the recent article by Triggiani et al (2017) reported in BJC. Their multicentre retrospective study adds to the existing body of knowledge-and controversy in the field-regarding the use of sterotactic body radiation therapy (SBRT) in the treatment of oligometastatic disease.

Their work provides meaningful information. Nevertheless, some aspects could be improved to produce more robust data. One such aspect is the number of patients included from the nine centres: four of the participating nine centres in their study enrolled a very small number of patients: $4-8$ patients. Another key aspect is short follow-up time (medium of 20, 4 months), a limitation already acknowledged by the authors.

There are also a few practical issues regarding biological effective dose (BED) we wish to add.

In this study, the radiation adopted schedules followed the local treatment policy of each centre, with patients treated with SBRT with a dose of at least $5 \mathrm{~Gy}$ per fraction to a biological effective dose of at least $80 \mathrm{~Gy}$, using an $\alpha / \beta$ of 3 .

When we calculated BED from the data presented in the above mentioned table, we found values ranging from $\mathrm{BED}_{3}=108 \mathrm{~Gy}(6 \mathrm{~Gy}$ per fraction/36 Gy total dose) to $\mathrm{BED}_{3}=180 \mathrm{~Gy}(12 \mathrm{~Gy}$ per fraction/36 Gy total dose). Results from several studies in oligometastatic disease have shown that biological effective dose (BED) represents an essential predictive factor of the efficacy of SBRT (reviewed in Kollar and Rengan, 2014; Ost et al, 2016). The authors did not find any significant correlation with distant progression-free survival and BED values. This may be explained in part due to the wide range of doses, fractionation schemes and heterogeneity of patient groups from the participating centres.

Finally, the authors chose an $\alpha / \beta$ ratio of 3 when using biological effective dose. Several experimental and clinical studies have suggested a relatively low $\alpha / \beta$ ratio for prostate cancer control $(\alpha / \beta=1,4$ ranging between 0.9 and $2.2 \mathrm{~Gy}$ ), lower than the corresponding $\alpha / \beta$ for lateresponding normal tissues (reviewed in Miralbell et al, 2012).

We obviously need more work in this area, with more pooled data analysis from SBRT prospective randomised clinical trials using various biologically effective dose (BED) and equivalent dose in 2 Gy (EQD2). In the next few years, we will have results from trials such as STOMP (the Surveillance or metastasis-directed Therapy for OligoMetastatic Prostate cancer recurrence) and CORE (the COnventional care or Radioablation in the treatment of Extracranial metastases) among others (reviewed in Stevens and Sooriakumaran, 2016). Evidence from these trials could be helpful, especially with longer follow-up times to help better optimise our treatment approaches, as called for by radiation oncologists (Lewis et al, 2017).

\section{CONFLICT OF INTEREST}

The author declares no conflict of interest.

\section{REFERENCES}

Kollar L, Rengan R (2014) Stereotactic body radiotherapy. Semin Oncol 41(6): 776-789.

Lewis S, Porceddu S, Nakamura N, Palma D, Lo S, Hoskin P, Moghakani D, Chmura S, Steven J (2017) Definitive stereotactic body radiotherapy (SBRT) for extracranial oligometastases: an international survey of $>1000$ radiation oncologists. Am J Clin Oncol 40(4): 418-422.

Miralbell R, Roberts S, Zubizarreta E, Hendry J (2012) Dose-fractionation sensitivity of prostate cancer deduced from radiotherapy outcomes of 5,969 patients in seven international institutional datasets: $\alpha / \beta=1.4(0.9-2.2)$ Gy. Int J Radiat Oncol Biol Phys 82(1): e17-e24.

Ost P, Jereczek-Fossa B, As N, Zilli T, Muacevic A, Olivier K, Henderson D, Casamassima F, Orecchia R, Surgp A, Brown L, Tree A, Mirabell R, De Meerleer G (2016) Progression-free survival following stereotactic body radiotherapy for oligometastatic prostate cancer treatment-naive recurrence: a multi-institutional analysis. Eur Urol 69(1): 9-12.

Stevens D, Sooriakumaran P (2016) Oligometastatic prostate cancer. Curr Treat Options Oncol 17: 62.

Triggiani L, Alongi F, Buglione M, Detti B, Santoni R, Bruni A, Maranzano E, Lohr F, D’Angelillo R, Magli A, Bonetta A, Mazzola R, Pasinetti N, Francolini G, Ingrosso G, Trippa F, Fersino S, Borghetti P, Ghirardelli P, Magrini SM (2017) Efficacy of stereotactic body radiotherapy in oligorecurrent and in oligoprogressive prostate cancer: new evidence from a multicentric study. Br J Cancer 116(12): 1520-1525.

This work is published under the BJC's standard license to publish agreement After 12 months the license terms will change to a Creative Commons AttributionNonCommercial-Share Alike 4.0 Unported License.

*Correspondence: Professor I Bravo; E-mail: isabel.bravo@ipoporto.min-saude.pt

Published online 16 November 2017

C 2018 Cancer Research UK. All rights reserved 0007 - 0920/18 